\title{
Theory of the Kondo lattice: competition between Kondo effect and magnetic order
}

\author{
B. COQBLIN*†, M. D. NÚNEZ-REGUEIRO†, A. THEUMANN†, \\ J. R. IGLESIAS $\$$ and S. G. MAGALHÃES§ \\ $\dagger$ Laboratoire de Physique des Solides Université Paris - Sud, \\ 91405 Orsay Cedex, France \\ fInstituto de Física - UFRGS Av. Bento Gonçalves 9500, \\ 91501-970 Porto Alegre, RS, Brazil \\ §Departamento de Fisica, Universidade Federal de Santa Maria, \\ 97105-900 Santa Maria, RS, Brazil
}

(Received 6 April 2005; in final form 1 June 2005)

\begin{abstract}
We present here a review of some theoretical features of the Kondo lattice problem, which can describe anomalous experimental properties of strongly correlated electron systems with mainly cerium, ytterbium or uranium. In such Kondo systems, there exist both a Kondo effect on each site and strong intersite magnetic interactions. We present firstly the general case of the Kondo lattice and the mean-field approximation. We then discuss the case of the "underscreened" Kondo lattice with a strong coexistence between the Kondo effect and a ferromagnetic order, as for example observed in uranium compounds such as UTe. We also discuss the Kondo-spin glass competition with eventually an additional magnetically ordered phase (ferromagnetic or antiferromagnetic one) and we can account for phase diagrams of some cerium and uranium disordered alloys, such as $\mathrm{CeNi}_{1-x} \mathrm{Cu}_{x}$. Finally, the introduction of a transverse magnetic field in the Kondo-spin glass problem yields a quantum critical point and allows a better description of the phase diagrams of disordered alloys.
\end{abstract}

\section{Introduction}

It is well established that an anomalous behaviour is observed in many cerium, ytterbium, uranium or other anomalous rare-earth (europium, samarium, praseodymium and thulium) and actinide (neptunium and plutonium) systems. These are different anomalous behaviours observed in such systems and a very important case concerns the "intermediate valence" systems, where the number of 4f electrons is generally not an integer and varies with the external pressure or the matrix concentration, like in the famous phase diagram of cerium metal [1-6] or in $\mathrm{Eu}\left(\mathrm{Pd}_{1-x} \mathrm{Au}_{x}\right)_{2} \mathrm{Si}_{2}$ alloys [7] or $\mathrm{YbInCu}_{4}$ [8]. Another interesting and well studied case concerns the so-called "Kondo system", which have a valence or a number of f-electrons close to an integer, but present however an anomalous

*Corresponding author. Email: coqblin@1ps.u-psud.fr 
behaviour due to a strong $\mathrm{d}-\mathrm{f}$ hybridization. The Kondo systems have been extensively studied experimentally and theoretically after the first explanation of the resistivity minimum of magnetic dilute alloys by Kondo [9] and the exact solution obtained within the renormalisation group technique for the single Kondo impurity [10].

In the case of lattice, there exists a strong competition between the Kondo effect and the magnetic order in many compounds or concentrated alloys containing rare-earths or actinides. This strong interplay between the Kondo effect on each site and the magnetic ordering arising from the Ruderman-Kittel-Kasuya-Yosida (RKKY) interaction between rare-earth atoms at different lattice sites is well described by the Doniach diagram [11], which gives the variation of the Néel temperature and of the Kondo temperature with increasing antiferromagnetic (AF) intrasite exchange interaction $J_{\mathrm{K}}$ between localized spins and conduction electron spins. It is well known that the ordering temperature $T_{\mathrm{N}}$ is increasing initially with increasing $\rho J_{\mathrm{K}}$, then passes through a maximum and tends to zero at a critical value $\rho J_{\mathrm{K}}^{\mathrm{c}}$ corresponding to a "quantum critical point" (QCP) in the Doniach diagram. Such a behaviour of $T_{\mathrm{N}}$ has been experimentally observed with increasing pressure in many cerium compounds, such as $\mathrm{CeAl}_{2}$ [12] or $\mathrm{CeRh}_{2} \mathrm{Si}_{2}$ [13]. A similar effect has been observed in $\mathrm{YbCu}_{2} \mathrm{Si}_{2}$ [14] or in related ytterbium compounds, where the Néel temperature starts from zero at a given pressure and increases rapidly with pressure, because pressure has an opposite effect in ytterbium compounds than in cerium compounds.

The Doniach diagram presents also the great advantage of separating cerium compounds in two categories: the first one includes cerium compounds which have a mixed Kondo-magnetic character corresponding to pressure below the critical pressure of the QCP; for example, $\mathrm{CeAl}_{2}$ has a Néel temperature of $3.8 \mathrm{~K}$ and also a relatively large electronic constant $\gamma$ of the specific heat equal to $135 \mathrm{~mJ} \mathrm{~mole}^{-1} \mathrm{~K}^{-2}$. The second category corresponds to cerium compounds with only a huge Kondo or heavy fermion character and for example $\mathrm{CeAl}_{3}$, which does not order magnetically, has an enormous value of $\gamma=1600 \mathrm{~mJ}$ mole ${ }^{-1} \mathrm{~K}^{-2}$. In the magnetically ordered compounds, there is coexistence between the magnetic order (generally AF like in $\mathrm{CeAl}_{2}$ ) and the heavy fermion character, although this effect is much smaller in magnetically ordered compounds than in nonmagnetic ones. On the other hand, the magnetic order can be AF, ferromagnetic in a few cases, of a classical spin glass (SG) order or a more disordered one.

The purpose of the present article is to review recent theoretical models, which can account for the difficult problem of competition or coexistence between the magnetic order and the Kondo effect, which gives the heavy fermion character. In the next section, we will firstly discuss the mean-field approximation and apply it to both the case of the Kondo effect in presence of short-range magnetic correlations and the case of the competition between the Kondo effect and the AF order. Then, we will develop the case of the so-called "underscreened" Kondo model where a coexistence between the Kondo effect and the magnetic order is obtained, which can account for the magnetic properties of some uranium compounds. Finally, we will discuss the occurrence of SG phases in cerium or uranium systems and we will describe the competition between the SG order, the Kondo effect and eventually a magnetically (ferromagnetic or AF) ordered phase. 


\section{The Kondo lattice model}

We have firstly developed the Kondo-lattice model, which is appropriate to describe cerium (ytterbium) compounds corresponding to a configuration close to $4 f^{1}$ (or $4 f^{13}$ ) with one $4 \mathrm{f}$ electron (or one hole). The Kondo-lattice model is often studied with only the intrasite Kondo interaction. In principle, both the RKKY magnetic interaction and the Kondo effect can be obtained from the intrasite exchange term [15]. When dealing with approximation schemes, it is hard to obtain both effects starting with the intrasite interaction alone and for this reason, an explicit intersite exchange interaction is usually included in the model Hamiltonian. We will give here a very brief summary of the work presented in $[16,17]$ and obviously details can be found in these articles.

The proposed Hamiltonian of the system is, therefore:

$$
H=\sum_{k \sigma} \varepsilon_{\mathrm{k}} n_{\mathrm{k} \sigma}^{c}+J_{\mathrm{K}} \sum_{i} \mathbf{s}_{i} \cdot \mathbf{S}_{i}+J_{\mathrm{H}} \sum_{\langle i j\rangle} \mathbf{S}_{i} \cdot \mathbf{S}_{j},
$$

where $\varepsilon_{\mathrm{k}}$ is the energy of the conduction band, $J_{\mathrm{K}}(>0)$ is the Kondo coupling between the localized spin $\mathbf{S}_{i}$ and the spin $\mathbf{s}_{i}$ of a conduction electron at the same site, and $J_{\mathrm{H}}$ is the interaction between nearest-neighbouring localized spins. Assuming spin-1/2 localized moments, we represented them by a zero-width $f$ band with one electron per site, while the conduction band has width $2 D$ and a constant density of states. We consider here the case of choosing $J_{\mathrm{H}}$ to be positive, implying that intersite interactions are $\mathrm{AF}$, as it is the case of most cerium compounds.

We now write the spin operators in fermionic representation and we impose the constraint of single-occupancy of the $f$ level at all sites, $n_{i}^{f}=1$.

In order to discuss the Kondo effect and magnetic correlations, we define the operators:

$$
\lambda_{i \sigma} \equiv c_{i \sigma}^{\dagger} f_{i \sigma}, \quad \Gamma_{i j \sigma} \equiv f_{i \sigma}^{\dagger} f_{j \sigma},
$$

where $\lambda_{i \sigma}$ describes the intrasite Kondo correlation and $\Gamma_{i j \sigma}$ represents an intersite correlation between two neighbouring atoms. This is so because the on-site spin-spin correlation $\left\langle\mathbf{s}_{i} \cdot \mathbf{S}_{i}\right\rangle$ can be written in terms of the average $\left\langle\lambda_{i \sigma} \lambda_{i,-\sigma}\right\rangle$ and similarly for the intersite correlation using $\Gamma_{i j \sigma}$. Then, with this notation we perform an extended mean-field approximation [15, 18], which is presented in full detail in $[16,17]$. Considering translational invariance and taking into account that there are no magnetic states, we can write $\lambda=\left\langle\lambda_{i \sigma}\right\rangle$ for all sites and $\Gamma=\left\langle\Gamma_{i j \sigma}\right\rangle$ for nearestneighbouring sites and zero otherwise. It is important to note that, in all the present mean-field descriptions of different Kondo lattice problems, we always use the same mean-field approximation, i.e. $\lambda=\left\langle\lambda_{i \sigma}\right\rangle$ in the nonmagnetic case or a spin-dependent $\lambda_{\sigma}=\left\langle\lambda_{i \sigma}\right\rangle$ in the magnetic case, for describing the Kondo problem, while we use different mean-field terms to describe the different parameters, like here the shortrange magnetic correlations or then different descriptions of the magnetic moments to study different cases of magnetic orderings. In fact, the mean-field approximation used here for the Kondo effect has the great advantage to describe simply and correctly the main features of the Kondo effect, but obviously it has the disadvantage of giving a too brutal mean-field-like transition rather than a continuous one. 
After performing this approximation, one deals with a one-electron Hamiltonian representing two hybridized bands: the conduction band of width $2 D$ and the $f$ band of effective band width $2 B D$, with $B$ given by:

$$
B=-\frac{z J_{\mathrm{H}} \Gamma}{D}
$$

$z$ being the number of nearest neighbours of a site.

The magnitude of the hybridization gap is directly related to $\lambda^{2}$ and this quantity is also a measure of the Kondo effect, as the Kondo correlation function $\left\langle\mathbf{s}_{i} \cdot \mathbf{S}_{i}\right\rangle$ is proportional to $\lambda^{2}$.

The mean-field parameters $\lambda$ and $\Gamma$ are obtained by minimizing the total internal energy of the system at zero temperature, or the total free energy at finite temperatures $[17,19]$. The reference energy $E_{0}$ of the $f$ band and the chemical potential $\mu$ have to be determined self-consistently in order to keep the average numbers of $f$ and the conduction electrons respectively equal to 1 and $n$.

We have previously treated the half-filled case, corresponding to a number of conduction electrons $n=1$ [16] and then the general case of a non-integer number of conduction electrons [17]. In this previous article, we have determined both the temperature $T_{\text {cor }}$ for the occurrence of short-range magnetic correlations and the Kondo temperature $T_{\mathrm{K}}$ for the lattice, which is different from the one-impurity Kondo temperature $T_{\mathrm{K} 0}$ obtained for $J_{\mathrm{H}}=0$.

In the limit $J_{\mathrm{H}}=0$ and for $J_{\mathrm{K}} / D \ll 1$, we obtain:

$$
T_{\mathrm{K}}=C_{0} D \sqrt{n(2-n)} \exp \left(-\frac{1}{\rho J_{\mathrm{K}}}\right)
$$

where $C_{0}$ is a numerical constant: $C_{0}=1.1337$. Equation (4) gives $T_{\mathrm{K}}$ depending on the band filling as $\sqrt{n(2-n)}$, which is consistent with a $\sqrt{n}$ dependence when $n \rightarrow 0$, as found in [20].

We have also extensively studied the variations of the Kondo temperature $T_{\mathrm{K}}$ with $J_{\mathrm{H}}$ and the conduction-band filling $n$ [17]. We have found that $T_{\mathrm{K}}$ first increases and then decreases with $J_{\mathrm{H}}$ for fixed $n$, dropping abruptly to zero at some critical value of $J_{\mathrm{H}}$. On the other hand, for a given $J_{\mathrm{H}}, T_{\mathrm{K}}$ decreases rapidly as $n$ departs from half filling. Thus, we have obtained a drastic suppression of the Kondo effect with increasing AF intersite coupling and reducing the band-filling factor. On the other hand, the correlation temperature $T_{\text {cor }}$ is linear with $J_{\mathrm{H}}$ independently of the value of $n$ and the short-range magnetic correlations occur above the Kondo temperature, in good agreement with neutron diffraction experiments in cerium systems, such as $\mathrm{CeCu}_{6}$ and $\mathrm{CeRu}_{2} \mathrm{Si}_{2}[21,22]$.

We have established that the dependence with the coupling constant $J_{\mathrm{K}}$ of the Kondo temperature for the lattice can be significantly different from the singleimpurity one [16, 17]. It is rather difficult to deduce experimentally the Kondo temperature especially in Kondo systems that are close to be magnetic and, therefore, to compare the preceding theoretical results with the experimental data. However, we can deduce from experimental results in $\mathrm{CeRh}_{2} \mathrm{Si}_{2}$ [13], $\mathrm{CeRu}_{2} \mathrm{Ge}_{2}$ [23, 24] 
or $\mathrm{Ce}_{2} \mathrm{Rh}_{3} \mathrm{Ge}_{5}$ [25] that the pressure dependence of $T_{\mathrm{K}}$ observed in these systems is different from the single-impurity case, in good agreement with our model.

But, on the other hand, the lattice Kondo temperature is closely following the single-impurity one in the other cerium and all ytterbium compounds. If we compare cerium and ytterbium compounds, the $4 \mathrm{f}$ electrons are more localized in the ytterbium case than in the cerium one and hence the intersite interaction $J_{\mathrm{H}}$ must be smaller in ytterbium systems than in cerium ones. Thus, in our present model, the pressure dependence of the lattice Kondo temperature must be closer to the single-impurity one in ytterbium compounds than in cerium ones, as experimentally observed. Further experiments are needed to better understand the conditions that can yield a Kondo temperature for the lattice much smaller than the singleimpurity one.

In this section, we have introduced the Kondo lattice problem, but in fact we have not accounted for the experimental curves of $T_{\mathrm{N}}$ versus pressure, which present a QCP. Thus, in order to better describe the behaviour of many cerium compounds, we have studied the competition between an AF order and the Kondo effect within the preceding model [26]. We use again the mean-field approximation by taking two order parameters, first $\lambda=\left\langle\lambda_{i \sigma}\right\rangle$ for describing the Kondo effect and then the average magnetization for each sub-lattice of the AF system. We have obtained a phase diagram showing a first-order transition from an AF order for small $J_{\mathrm{K}}$ values to a Kondo phase for larger $J_{\mathrm{K}}$ values. The transition at low temperatures between the AF phase to the Kondo one with increasing $J_{\mathrm{K}}$ (or equivalently increasing pressure in Ce compounds) is a first order one without any QCP and we have, therefore, obtained a competition between these two phases and not a coexistence between them, as observed in many cerium compounds, such as $\mathrm{CeAl}_{2}$. This mean-field model does not give any "mixed" AF-Kondo phase, but is able to give successively an AF order and a Kondo phase with increasing $\left|J_{\mathrm{K}}\right|$.

Recently, the competition between the ferromagnetic order and the Kondo effect has been studied within the same Kondo lattice problem using the same mean-field approximation [27, 28]. The ferromagnetic order exists even for $J_{\mathrm{H}}=0$ and the magnetic moment is maximum for small $J_{\mathrm{K}}$ values. The Kondo order parameter $\lambda$ increases and the magnetic moment decreases with increasing $J_{\mathrm{K}}$, but however it remains an interval for $J_{\mathrm{K}}$ where there exists a coexistence between the ferromagnetic order and the Kondo effect. This effect is relatively small with respect to the large coexistence, which is obtained for the underscreened Kondo lattice as we will see in the next section.

\section{The underscreened Kondo lattice model}

As we have seen in the previous section, there is a strong competition between the Kondo effect and the magnetic order at low temperature in cerium and ytterbium compounds [17]. On the other hand, the situation is different in uranium systems. Uranium compounds, such as $\mathrm{UBe}_{13}$ [29], $\mathrm{URu}_{2} \mathrm{Si}_{2}$ [30], $\mathrm{UNi}_{2} \mathrm{Al}_{3}$ and $\mathrm{UPd}_{2} \mathrm{Al}_{3}$ $[31,32]$ present a heavy fermion behaviour characterized by large values of the electronic specific heat constant, then order antiferromagnetically, and undergo 
finally a transition to a superconducting state, as temperature decreases. The situation of $\mathrm{UPt}_{3}$ is even more complex, because probably short-range magnetic correlations rather than a normal AF order exist [33, 34]. Finally, UTe is a dense Kondo system undergoing a ferromagnetic ordering at the large Curie temperature of $T_{\mathrm{c}}=102 \mathrm{~K}$ [35]. We can conclude that there is a strong coexistence between the heavy fermion character and the magnetic order in U-compounds rather than the competition generally invoked in $\mathrm{Ce}$ - or Yb-compounds.

Cerium and ytterbium Kondo compounds are well described by the regular Kondo effect corresponding to the $4 f^{1}$ and $4 f^{13}$ configurations, while uranium ions in such compounds have a configuration $5 f^{n}$, with $n=2$ or 3 . The dual nature of the $5 f$ electrons and the possible coexistence of localized and itinerant $5 f$ states has been developed experimentally [36] and theoretically [37] to explain behaviour of U-compounds.

We present here a theoretical model to describe the "underscreened Kondo-lattice" treated in the mean-field approximation [38]. This model describes a lattice of ions in the $5 f^{2}$ configuration with a $5 f$-spin $S=1$, in Kondo-like interaction with classical conduction electrons with $s=1 / 2$ corresponding, therefore, to an underscreened situation for the $f$-electrons. The underscreened Kondo effect has been solved exactly for the single-impurity case [39] and studied for the case of two $S=1$ impurities [40].

To describe the mean-field approximation for the underscreened Kondo model, the $S=1$ localized spins are decomposed into two $1 / 2$ spins $\tau_{i \sigma}^{f n}$. We impose the condition that these two spins are always ferromagnetically aligned and we take only the two $z$-components +1 and -1 of each spin $S=1$ in order to have a fermionic description of the problem with only four fermionic operators [40].

Thus, the lattice Hamiltonian is given here by:

$$
\begin{aligned}
H= & \sum_{k, \sigma} \varepsilon_{k} n_{k \sigma}^{c}+\sum_{i \sigma} E_{0}\left(n_{i \sigma}^{f 1}+n_{i \sigma}^{f 2}\right)+J_{\mathrm{K}} \sum_{i \sigma} \vec{s}_{i}^{c}\left(\vec{\tau}_{i \sigma}^{f 1}+\vec{\tau}_{i \sigma}^{f 2}\right) \\
& +J_{\mathrm{H}} \sum_{i, j}\left(\vec{\tau}_{i \sigma}^{f 1}+\vec{\tau}_{i \sigma}^{f 2}\right)\left(\vec{\tau}_{j \sigma}^{f 1}+\vec{\tau}_{j \sigma}^{f 2}\right)
\end{aligned}
$$

where the first term describes the conduction band, the second term the two-fold degenerate $f$-band without width, the third term the intra-site Kondo interaction (with $J_{\mathrm{K}}>0$ ) and the fourth term the inter-site interaction between $f$-magnetic moments.

In fact, a more complete representation will be to take a representation of the $S=1$ spins with the three components $+1,-1$ and 0 . We are presently studying the problem with this full quantum description [41].

Then here, we use the mean-field approximation and expand the proceeding Hamiltonian according to the following four mean-field values:

$$
\begin{aligned}
M & =\left\langle S_{i}^{z}\right\rangle=\left\langle\tau_{i z}^{f 1}+\tau_{i z}^{f 2}\right\rangle \\
m & =\left\langle s_{i}^{c s}\right\rangle \\
\lambda_{\sigma} & =\sum_{n}\left\langle f_{i \sigma n}^{+} c_{i \alpha}\right\rangle
\end{aligned}
$$

$\lambda_{\sigma}$ is taken independent of the site $i$ and of $n=1,2$, but is spin-dependent. 
The resulting bands are one non-hybridized $f$-band at energy $E_{0}+\sigma J_{\mathrm{K}} m / 2+$ $\sigma J_{\mathrm{H}} M z / 4$ and two $k-f$ hybridized bands with spin-dependent energy:

$$
\begin{aligned}
E_{ \pm}^{\sigma}(k)= & \frac{1}{2}\left(\varepsilon_{k}+E_{0}+\frac{\sigma J_{\mathrm{K}}(M+m)}{2}+\frac{\sigma J_{\mathrm{H}} M z}{4}\right) \\
& \pm \frac{1}{2} \sqrt{\left(\varepsilon_{k}-E_{0}+\frac{\sigma J_{\mathrm{K}}(M-m)}{2}-\frac{\sigma J_{\mathrm{H}} M z}{4}\right)^{2}+2 J_{\mathrm{K}}^{2} \lambda_{-\sigma}^{2}}
\end{aligned}
$$

where $\sigma= \pm 1$ for the two spins $\uparrow$ and $\downarrow$ and $z$ is the number of neighbouring atoms. The bands for $\uparrow$ and $\downarrow$ spins are shifted from each other. The resulting hybridization is double here than in the $S=1 / 2 f$-spin case.

Then, the equations giving $M, m, \lambda_{\uparrow}$ and $\lambda_{\downarrow}$ are obtained by taking the number of $f$-electrons $N_{f}=\Sigma_{\sigma}\left(n_{\sigma}^{f 1}+n_{\sigma}^{f 2}\right)=2$ and a fixed number of conduction electrons $n=\Sigma_{\sigma}\left\langle n_{\sigma}^{\mathrm{c}}\right\rangle$. The magnetic moment $M$ arises from $f$-electrons and $m$ from conduction electrons. $E_{0}$ is also expressed as a function of the four variables. The solutions for $M, m, \lambda_{\uparrow}$ and $\lambda_{\downarrow}$ are obtained for the Kondo (which corresponds to $J_{\mathrm{K}}>0$ ) and ferromagnetic (which corresponds to $J_{\mathrm{H}}<0$ ) case at $T=0$ by solving the self-consistent equations.

In conclusion, we emphasize the two following points: first, we obtain solutions with both a magnetization $(M \neq 0, m \neq 0)$ coming mostly from the non-hybridized hand and a Kondo character $\left(\lambda_{\uparrow}\right.$ and $\left.\lambda_{\downarrow} \neq 0\right)$; this result can account for the coexistence between magnetic order and heavy fermion character observed in uranium compounds, such as UTe. Second, we study the influence of the different parameters like $J_{\mathrm{H}}$ which tends to give the ferromagnetic order and $J_{\mathrm{K}}$ which favours the Kondo effect. A full description of the results will be published elsewhere [41, 42].

\section{The Kondo lattice-spin glass competition}

Above the QCP, there exists a very strong heavy fermion character, but several possible behaviours, i.e. the classical Fermi liquid one with eventually a reduced Kondo temperature $[13,17]$ or different Non-Fermi-Liquid (NFL) ones, have been observed in cerium or ytterbium compounds [43-45].

On the other hand, the disorder can yield a SG phase in addition to the Kondo (mainly NFL) behaviour at low temperatures around the QCP in disordered cerium or uranium alloys. This is the case of the magnetic phase diagram of $\mathrm{CeNi}_{1-} \mathrm{Cu}_{x}$ alloys that have been extensively studied [46, 47], while the phase diagram of $\mathrm{Ce}_{2} \mathrm{Au}_{1-x} \mathrm{Co}_{x} \mathrm{Si}_{3}$ alloys presents the sequence of $\mathrm{SG}-\mathrm{AF}-\mathrm{Kondo}$ phases at low temperatures with increasing cobalt concentration [48]. The three phases, AF, SG and NFL have been obtained at low temperatures for different concentrations in $\mathrm{UCu}_{5-x} \mathrm{Pd}_{x}$ [49] or in $\mathrm{U}_{1-x} \mathrm{La}_{x} \mathrm{Pd}_{2} \mathrm{Al}_{3}$ [50] disordered alloys. Thus, a SG-Kondo transition has been observed experimentally with increasing concentration in disordered alloys around the QCP. Recent experiments performed in $\mathrm{CeNi}_{1-x} \mathrm{Cu}_{x}$ alloys have shown that the phase diagram of these alloys is more complicated [51, 52]. In fact, when temperature decreases, for alloys with $0.7>x>0.1$, there appears 
a "cluster spin glass" phase presenting magnetic inhomogeneities, rather than a classical SG phase. At lower temperatures, there is a percolative process, which increases the size of the clusters, to finally yield a ferromagnetic phase at very low temperatures. On the other hand, the transition to the Kondo phase when $x$ decreases below roughly 0.15 is very smooth without any QCP and it seems that some magnetic clusters remain at very low temperatures with $x$ approaching zero.

The transition between a SG phase and a Kondo one with, in addition, the presence of a magnetically ordered (ferromagnetic or AF) phase, has been recently studied in detail in the case of Kondo lattices. The SG-Kondo transition was firstly studied theoretically [53], then in the presence of ferromagnetic ordering [54] or AF ordering [55] and finally the model has been improved in order to obtain a good description of the QCP [56], by introducing a transverse magnetic field.

In the first article on SG-Kondo phase transitions, we take a Hamiltonian that couples the localized spins of a Kondo lattice with an additional long range random interaction, like in the Sherrington-Kirkpatrick SG model [57]. The localized spins of the Kondo lattice, with localized spins $\vec{S}_{i}$, interact with conduction electrons and the Kondo effect is studied in the previously described mean-field decoupling scheme $[15,17]$. The $\mathrm{SG}$ interaction is described by the quantum Ising Hamiltonian where only interact the $z$-components of the localized spins [58-60].

The Hamiltonian of the model is:

$$
\mathcal{H}=\sum_{k, \sigma} \varepsilon_{\mathrm{k}} n_{\mathrm{k} \sigma}+\varepsilon_{0} \sum_{i, \sigma} n_{i \sigma}^{f}+J_{\mathrm{K}} \sum_{i}\left[S_{f i}^{+} s_{c i}^{-}+S_{f i}^{-} s_{c i}^{+}\right]-\sum_{i, j} J_{i j} S_{f i}^{z} S_{f j}^{z}
$$

where, as usual, $J_{\mathrm{K}}>0$.

The localized and conduction electron spins are then written in terms of fermion operators $f_{i \sigma}$ or $c_{i \sigma}$. The energies $\varepsilon_{0}\left(\varepsilon_{\mathrm{k}}\right)$ are referred to the chemical potentials $\mu_{f}\left(\mu_{c}\right)$, respectively.

To describe the nature of the SG, the coupling $J_{i j}$ is taken here as an independent random variable with the gaussian distribution:

$$
\mathcal{P}\left(J_{i j}\right)=\frac{1}{J} \sqrt{\frac{N}{64 \pi}} \exp \left\{-\frac{\left(J_{i j}+2 J_{0} / N\right)^{2}}{64 J^{2}} N\right\} .
$$

The preceeding expression represents the random distribution of the exchange integrals $J_{i j}$. The average value $J_{0}$ has been taken equal to zero to describe the SG-Kondo transition [53], while the case $J_{0}<0$ produces a complex phase diagram with SG, ferromagnetic, mixed (a SG with spontaneous magnetization) and Kondo phases [54]. Finally, the case $J_{0}>0$ corresponds to an AF phase in competition with SG and Kondo phases [55].

The Kondo and SG transitions are both described within the mean-field approximation. Using functional integrals techniques and a static, replica symmetric Ansatz for the Kondo and SG order parameters, a mean-field expression for the free energy and the saddle point equations for the order parameters have been derived. Numerical solutions of the saddle-point equations allow us to determine the Kondo order parameter $\lambda$ and the SG parameter $q$.

In the first studied case [53] with $J_{0}=0$, we obtain three phases: at high temperatures, the "normal" phase is paramagnetic with $\lambda=q=0$. When the temperature 
is lowered, for not too large values of the ratio $J_{\mathrm{K}} / J$, a SG phase, with $q>0$ and $\lambda=0$, is obtained and, for large values of the ratio $J_{K} / J$, we recover the "Kondo" phase with a non-zero $\lambda$ value and $q=0$. The transition line from the SG phase to the Kondo phase is a first-order one and ends at $J_{\mathrm{K}}^{\mathrm{c}}$ at $T=0$. When the temperature is lowered, the transition temperature does not vary very much with the value of $J_{\mathrm{K}} / J$; the separation between the SG and the Kondo phases deviates completely from the behaviour of the single-impurity Kondo temperature $T_{\mathrm{K}}$ and looks like the separation between the AF and Kondo phases when these two phases are considered [26]. We can also remark that we get here only "pure" Kondo or SG phases and never a mixed SG-Kondo phase with the two order parameters different from zero; this result is probably connected to the approximations used here to treat the starting Hamiltonian.

The diagram obtained theoretically for the SG-Kondo transition can give a rough account for the magnetic phase diagram observed above the Curie temperature for the $\mathrm{CeNi}_{1-x} \mathrm{Cu}_{x}$ [46] for small $x$ values when there is a transition from a SG state to a Kondo state and then to the intermediate valence compound CeNi. However, the theoretical model gives an average description, while recent experiments have shown the existence of magnetic clusters and our model cannot be checked from that point of view $[51,52]$. There is also probably a SG-Kondo transition in $\mathrm{CeCoGe}_{3-x} \mathrm{Si}_{x}$ alloys, but there the experimental situation is also very complicate [61].

Indeed the experimental situation is generally more complex in disordered cerium or uranium alloys and there generally exists an antiferromagnetically ordered phase in addition to the SG phase and to the Kondo one, which moreover has a NFL behaviour close to the QCP. For example, the three phases, AF, SG, and NFL have been obtained at low temperatures for different concentrations in $\mathrm{UCu}_{5-} \mathrm{Pd}_{x}$ [49] or in $\mathrm{U}_{1-x} \mathrm{La}_{x} \mathrm{Pd}_{2} \mathrm{Al}_{3}$ [50] disordered alloys. The sequence AF-NFL-SG occurs with increasing $x$ in $\mathrm{UCu}_{5-x} \mathrm{Pd}_{x}$ [49] and the opposite sequence $\mathrm{AF}-\mathrm{SG}-\mathrm{NFL}$ with increasing $x$ in $\mathrm{U}_{1-x} \mathrm{La}_{x} \mathrm{Pd}_{2} \mathrm{Al}_{3}[50]$.

The two theoretical cases SG-ferromagnetic-Kondo [54] and SG-AF-Kondo [55] have been studied within the same type of mean-field approximation, but here there are at least three order parameters, the SG order parameter $q$, the magnetization $M$ (or the magnetization of each sub-lattice in the AF case) and finally the Kondo parameter $\lambda$, which is generally taken as spin independent. The calculation is even more difficult in the AF case, because we are obliged to consider two sub-lattices with opposite magnetizations. We present here the results obtained for the $\mathrm{SG}-\mathrm{AF}-\mathrm{K}$ ondo phase sequence with a positive value of $J_{0}$. There are three parameters $J_{\mathrm{K}}, J_{0}$ and $J$, but actually, $J_{\mathrm{K}}$ cannot be considered as independent of $J_{0}$ and $J$, since an intrasite exchange term would yield both a Kondo effect and RKKY interaction. This problem has been already discussed and we have taken the two possible choices: either we take the values of the three parameters independent from each other; or we adopt some conjecture relating $J_{\mathrm{K}}, J_{0}$ and $J$. In our theoretical approach, the variance $J$ is kept constant and we take the following relationship between $J_{0}$ and $J_{\mathrm{K}}$ :

$$
\frac{J_{0}}{J}=\alpha\left(\frac{J_{\mathrm{K}}}{J}\right)^{2} .
$$




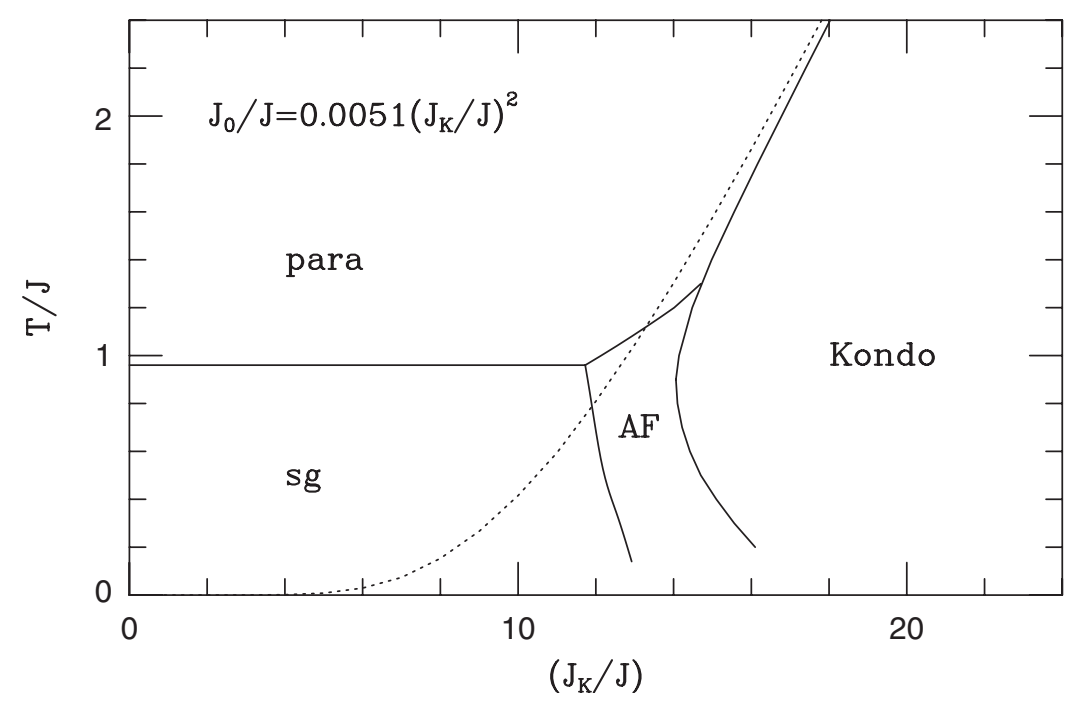

Figure 1. The phase diagram $T / J$ versus $J_{\mathrm{K}} / J$ showing the sequence of spin glass (SG), antiferromagnetism (AF) and Kondo phases. The variance $J$ is kept constant. The dotted line denotes the "pure" (i.e. single-impurity) Kondo temperature.

The quadratic choice in equation (10) is taken here in order to have a rough simulation of the fact that the RKKY interaction behaves as $J_{\mathrm{K}}^{2}$, as already assumed in [16]. A phase diagram obtained for a typical value $\alpha=0.0051$ is shown in figure 1.

The numerical solutions of the order parameters yield the sequence of phases $\mathrm{SG}-\mathrm{AF}-\mathrm{Kondo}$ shown in figure 1 . The Kondo temperature $T_{\mathrm{K}}$ is decreasing with decreasing values of $J_{\mathrm{K}} / J$ down to $J_{\mathrm{K}} / J \approx 15$. From this point onwards, there is a phase transition leading to an AF order with the Néel temperature $T_{\mathrm{N}}$ monotonically decreasing with $J_{\mathrm{K}} / J$. For still smaller values of $J_{\mathrm{K}} / J$ (and therefore smaller values of $\left.J_{0} / J\right)$, the SG behaviour becomes dominant.

Finally, figure 1 can roughly account for the phase diagram of $\mathrm{Ce}_{2} \mathrm{Au}_{1-x} \mathrm{Co}_{x} \mathrm{Si}_{3}$ alloys, which presents the sequence of $\mathrm{SG}-\mathrm{AF}-\mathrm{K}$ ondo phases at low temperatures with increasing cobalt concentration [48]. However, in contrast to our theoretical model, the experimental AF phase ends at a critical point before reaching the Kondo phase when the cobalt concentration $x$ is still increasing.

Thus, in order to improve the theoretical description of the SG-Kondo or SG-AF-Kondo transitions and to obtain a good description of the QCP, a new model has been developed by adding a transverse field to the previous Hamiltonian [56]. In fact, in previous publications the resultant RKKY interaction was introduced by means of random, infinite range couplings among the $S^{z}$ components of the localized spins as in the Sherrington-Krikpatrick model [57] and by neglecting the spin flip coupling of the transverse components. The previous models failed to describe a second-order QCP at $T=0$, because, by disregarding the spin flipping part of the Heisenberg Hamiltonian, we suppressed the tunneling mechanism and magnetic ordering occurs only along the $z$-axis. In order to introduce a spin flipping mechanism and to avoid the intricacies of the random Heisenberg model, 


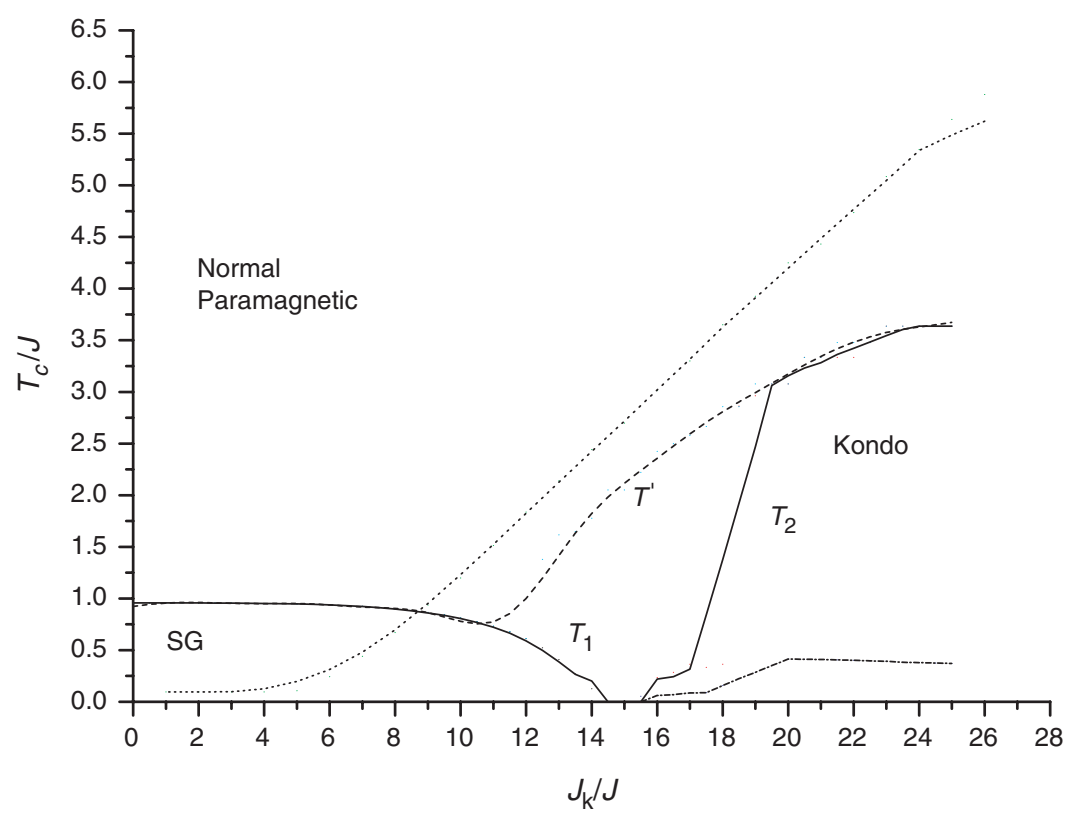

Figure 2. Phase diagram in the $T-J_{\mathrm{K}}$ plane as a function of $T / J$ and $J_{\mathrm{K}} / J$ for the transverse field $\Gamma=\alpha J_{\mathrm{K}}^{2}$, for fixed values of $J$ and $D$, with $D / J=12$, and for two values $\alpha J=0.01348$ (solid lines) and $\alpha J=0.01344$ (dashed line). The critical (solid) second-order line $T_{1}$ for low values of $J_{\mathrm{K}}$ separates the paramagnetic phase $(q=\lambda=0)$ for high temperatures from the SG phase $(q \geq 0, \lambda=0)$ at low temperatures and ends at a QCP. The critical second-order solid line $T_{2}$ for large values of $J_{\mathrm{K}}$ separates the paramagnetic phase from the Kondo phase $(\lambda \geq 0, q=0)$ and ends at a second QCP. The dotted line represents the "pure" (i.e. singleimpurity) Kondo temperature $T_{\mathrm{K}}$ and the dashed-dotted line represents the residual magnetic moment $\bar{\chi}_{\mathrm{c}}\left(J_{\mathrm{K}}\right)$ on the line $T=T_{2}$. The critical line (dashed) labeled by $T^{\prime}$ corresponds to $\alpha J=0.01344$ and has no QCP.

the Heisenberg-like coupling among the three spin components induced by the RKKY interaction is mimicked by a quantum Ising SG in a transverse field. Thus, the Hamiltonian of the model is taken here as the sum of the Hamiltonian given by equation (8) and of an additional term given by:

$$
\mathcal{H}_{x}=-2 \Gamma \sum_{i} S_{f i}^{x}
$$

The coupling $J_{i j}$ in equation (8) is described by a gaussian distribution of zero mean $J_{0}$ as previously used for the SG-Kondo transition [53].

Numerical solutions of the saddle-point equations allow us to draw in figure 2 the magnetic phase diagram in the $J_{\mathrm{K}}$ versus $T$ plane, using the relation $\Gamma=\alpha J_{\mathrm{K}}^{2}$ between the transverse field and $J_{\mathrm{K}}$, fixed values of $J$ and $D$, with $D / J=12$ and a value of the parameter $\alpha J: \alpha J=0.01348$ [56]. Figure 2 shows three different phases: at high temperatures, the "normal" phase is paramagnetic with vanishing Kondo and SG order parameters, i.e. $\lambda=q=0$. When the temperature is lowered, for not too large values of the ratio $J_{\mathrm{K}} / J$, a second-order transition line is found at $T=T_{1}$ to 
a SG phase with $q>0$ and $\lambda=0$. The critical line $T_{1}\left(J_{\mathrm{K}}\right)$ ends at a QCP $J_{\mathrm{K} 1}^{\mathrm{c}}$. Then, for larger values of the ratio $J_{\mathrm{K}} / J$, there is a second-order transition line starting at $J_{\mathrm{K} 2}^{\mathrm{c}}$ to a Kondo state with $\lambda \geq 0$. These results are very sensitive to the value of $D / J$ and $\alpha J$. For $D / J=12$ and for example $\alpha J=0.01344$, the two QCP disappear, as shown in figure 2 , where the new transition is given by the dashed curve labeled $T^{\prime}$. Thus, the occurrence of two QCP is extremely sensitive to the precise value of $\alpha J$. However, the phase diagram shown in figure 2 with two very close QCP improves considerably the theoretical description of the SG-Kondo transition [56]. We can also remark that we get here only "pure" Kondo or SG phases and never a mixed SG-Kondo phase with the two order parameters different from zero, as already noticed in our mean-field calculations [26].

A QCP has been observed experimentally in several cerium and uranium disordered alloys [48-50] and the phase diagram shown in figure 2 improves considerably the description of the SG-Kondo transition with respect to [53]. However, the experimental situation appears to be really complicate, as shown for example by recent experiments on $\mathrm{CeNi}_{1-x} \mathrm{Cu}_{x}$ alloys [51, 52]. Thus, further experimental work is certainly necessary, but however the models presented here can account roughly for the behaviour of heavy fermion disordered alloys, especially in the vicinity of the QCP.

\section{Conclusions}

We have presented here a brief review on recent works on the Kondo lattice problem. We have always used here the same mean-field approximation for the Kondo effect, which is simple and gives correct results, but yields however a too brutal (typically mean-field-like) transition rather than the expected continuous one around the Kondo temperature. Here, we have firstly considered the case of cerium (ytterbium) compounds corresponding to a configuration close to $4 f^{1}$ (or $4 f^{13}$ ) with one $4 \mathrm{f}$ electron (or one hole) and then the case of uranium described here by a configuration close to $5 f^{2}$. The "normal" Kondo effect describes the first case, while the "underscreened" Kondo effect is more appropriate for the second one. Finally, we have discussed the interaction between the Kondo effect and a disordered phase that we describe as a SG phase, but in fact the SG-Kondo problem is more complicate, as shown for example by recent experiments on $\mathrm{CeNi}_{1-x} C u_{x}$ alloys, which have yielded a new "cluster spin glass". Finally, further experimental results and a more sophisticated theoretical approach, eventually beyond our mean-field approximation, are necessary to improve the description of anomalous rare-earth and actinide systems.

\section{References}

[1] A. Jayaraman, Phys. Rev. 137179 (1965).

[2] B. Coqblin and A. Blandin, Adv. in Phys. 17281 (1968).

[3] R. Ramirez and L.M. Falicov, Phys. Rev. B 32425 (1971).

[4] J.W. Allen and R.M. Martin, Phys. Rev. Lett. 491106 (1982).

[5] M. Lavagna, C. Lacroix and M. Cyrot, J. Phys. F 131007 (1983). 
[6] A. Svane, Phys. Rev. B 534275 (1996).

[7] C.U. Segre, M. Croft, J.A. Hodges, V. Murgai, L.C. Gupta and R.D. Parks, Phys. Rev. Lett. 491947 (1982).

[8] J.L. Sarrao, Physica B 259-261 128 (1999).

[9] J. Kondo, Progr. Theoret. Phys. (Kyoto) 3237 (1964).

[10] K.G. Wilson, Rev. Mod. Phys. 47773 (1975).

[11] S. Doniach, in Proceedings of the International Conference on Valence Instabilities and Related Narrow-Band Phenomena, edited by R.D. Parks (Plenum Press: New York; 1976), p. 168.

[12] B. Barbara, H. Bartholin, D. Florence, M.F. Rossignol and E. Walker, Physica B 86-88 188 (1977).

[13] T. Graf, J.D. Thompson, M.F. Hundley, R. Movshovich, Z. Fisk, D. Mandrus, R.A. Fischer and N.E. Phillips, Phys. Rev. Lett. 783769 (1997).

[14] K. Alami-Yadri, H. Wilhelm and D. Jaccard, Physica B 259-261 157 (1999).

[15] C. Lacroix and M. Cyrot, Phys. Rev. B 201969 (1979).

[16] J.R. Iglesias, C. Lacroix and B. Coqblin, Phys. Rev. B 5611820 (1997).

[17] B. Coqblin, C. Lacroix, M.A. Gusmao and J.R. Iglesias, Phys. Rev. B 67066617 (2003).

[18] P. Coleman and N. Andrei, J. Phys.: Condens. Matter 14057 (1989).

[19] A.R. Ruppenthal, J.R. Iglesias and M.A. Gusmao, Phys. Rev. B 607321 (1999).

[20] S. Burdin, A. Georges and D.R. Grempel, Phys. Rev. Lett. 851048 (2000); S. Burdin, thesis, Le reseau Kondo a basse temperature: du liquide de Fermi au liquide de spin, Grenoble (2001).

[21] J. Rossat-Mignod, L.P. Regnault, J.L. Jacoud, C. Vettier, P. Lejay, J. Flouquet, E. Walker, D. Jaccard and A. Amato, J. Mag. Mag. Mater. 76-77 376 (1988).

[22] L.P. Regnault, W.A.C. Erkelens, J. Rossat-Mignod, P. Lejay and J. Flouquet, Phys. Rev. B 384481 (1988).

[23] S. Sullow, M.C. Aronson, B.D. Rainford and P. Haen, Phys. Rev. Lett. 822963 (1999).

[24] H. Wilhelm, K. Alami-Yadri, B. Revaz and D. Jaccard, Phys. Rev. B 593651 (1999).

[25] K. Umeo, T. Takabatake, T. Suzuki, S. Hane, H. Mitamura and T. Goto, Phys. Rev. B 64144412 (2001).

[26] B. Coqblin, M.A. Gusmao, J.R. Iglesias, A.R. Ruppenthal and C. Lacroix, J. Magn. Magn. Mater. 226-230 115 (2001).

[27] Mun Dae Kim, Chul Koo Kim and Jongbae Hong, Phys. Rev. B 68174424 (2003).

[28] M.D. Nunez-Regueiro and B. Coqblin, in Proceedings of SCES'04 Conference, Physica B 359-361 741 (2005).

[29] R.N. Kleiman, D.J. Bishop, H.R. Ott, Z. Fisk and J.L. Smith, Phys. Rev. Lett. 64 1975 (1990).

[30] T.T.M. Palstra, A.A. Menovsky, J. van den Berg, A.J. Dirkmaat, P.H. Kes, G.J. Nieuwenhuys and J.A. Mydosh, Phys. Rev. Lett. 552727 (1985).

[31] C. Geibel, S. Thies, D. Kaczorowski, A. Mehner, A. Grauel, B. Seidel, U. Ahlheim, R. Helfrich, K. Petersen, C.D. Bredl and F. Steglich, Z. Phys. B 83305 (1991).

[32] C. Geibel, C. Schank, S. Thies, H. Kitazawa, C.D. Bredl, A. Bohm, M. Rau, A. Grauel, R. Caspary, R. Helfrich, U. Ahlheim, G. Weber and F. Steglich, Z. Phys. B 841 (1991).

[33] A. de Visser, R.J. Keizer, A.A. Menovsky, M. Mihalik, F.S. Tautz, J.J.M. Franse, B. Fak, N.H. van Dijk, J. Flouquet, J. Bossy and S. Pujol, Physia B 230-232 49 (1997).

[34] A. de Visser, R.J. Keizer, R. van Harrevelt, A.A. Menovsky, J.J.M. Franse, A. Amato, F.N. Gygax, M. Pinkpank and A. Schenck, Physica B 230-232 53 (1997).

[35] J. Schoenes, J. Less-Common Metals 12187 (1986).

[36] F. Steglich, P. Gegenwart, C. Geibel, R. Helfrich, P. Hellmann, M. Lang, A. Link, R. Modler, G. Sparn, N. Buttgen and A. Loidl, Physica B 223-224 1 (1996).

[37] G. Zwicknagl, A.N. Yaresko and P. Fulde, Phys. Rev. B 65081103 (2002).

[38] M.D. Nunez-Regueiro, B. Coqblin and J.R. Iglesias, J. Magn. Magn. Mater. 272-276 e95 (2004).

[39] P. Schlottman and P.D. Sacramento, Adv. in Phys. 42641 (1993).

[40] Karyn Le Hur and B. Coqblin, Phys. Rev. B 56668 (1997). 
[41] N. Perkins, M.D. Nunez-Regueiro, J.R. Iglesias and B. Coqblin, in Proceedings of Vienna SCES'05 Conference, Physica B (2006).

[42] N. Perkins, M.D. Nunez-Regueiro, J.R. Iglesias and B. Coqblin, to be published.

[43] B. Coqblin, M.A. Gusmao, J.R. Iglesias, A. Theumann, C. Lacroix, S.G. Magalhaes and A.A. Schmidt, Acta Phys. Polonica 341273 (2003).

[44] P. Coleman, Physica B 259-261 353 (1999).

[45] E. Miranda and V. Dobrosavljevic, Phys. Rev. Lett. 86264 (2001).

[46] J. Garcia Soldevilla, J.C. Gomez Sal, J.A. Blanco, J.I. Espeso and J. Rodriguez Fernandez, Phys. Rev. B. 616821 (2000).

[47] J.C. Gomez Sal, J. Garcia Soldevilla, J.A. Blanco, J.I. Espeso, J. Rodriguez Fernandez, F. Luis, F. Bartolome and J. Bartolome, Phys. Rev. B 5611741 (1997).

[48] S. Majumdar, E.U. Sampathkumarar, St. Berger, M. Della Mea, H. Mickor, M. Brando, J. Hemberger and A. Loidl, Solid State Comm. 121665 (2002).

[49] R. Vollmer, T. Pietrus, H.V. Löneyssen, R. Chan and M.B. Maple, Phys. Rev. B 611218 (2000).

[50] V.S. Zapf, R.P. Dickey, E.J. Freeman, C. Sirvent and M.B. Maple, Phys. Rev. B 65024437 (2001).

[51] N. Marcano, G.M. Kalvius, D.R. Noakes, J.C. Gomez-Sal, R. Wappling, J.I. Espeso, E. Schreier, A. Kratzer, Ch. Baines and A. Amato, Physica Scripta 201 (2003).

[52] N. Marcano, J.I. Espeso, J.C. Gomez-Sal, J. Rodriguez Fernandez, J. Herrero-Albellos and F. Bartolome, Phys. Rev. B 71134401 (2005): N. Marcano, thesis, In homogeneidades intrinsecas en el sistema de electrones Fuertemente correlacionados CeNi1-xCux, University of Cantabria (2005).

[53] Alba Theumann, B. Coqblin, S.G. Magalhaes and A.A. Schmidt, Phys. Rev. B 63054409 (2001).

[54] S.G. Magalhaes, A.A. Schmidt, Alba Theumann and B. Coqblin, Eur. Phys. J. B 30 419 (2002).

[55] S.G. Magalhaes, A.A. Schmidt, F.M. Zimmer, Alba Theumann and B. Coqblin, Eur. Phys. J. B 34447 (2003).

[56] Alba Theumann and B. Coqblin, Phys. Rev. B 69214418 (2004).

[57] D. Sherrington and S. Kirkpatrick, Phys. Rev. Lett. 351792 (1975).

[58] Anirvan M. Sengupta and Antoine Georges, Phys. Rev. B 5210295 (1995).

[59] Alba Theumann and M. Vieira Gusmao, Phys. Lett. 105A 311 (1984).

[60] S.G. Magalhaes and Alba Theumann, Eur. Phys. J. B 95 (1999).

[61] D. Eom, M. Ishikaw, J. Kitagawa and N. Takeda, J. Phys. Soc. Japan 672495 (1998). 